\title{
Large particle segregation in two-dimensional sheared granular flows
}

\author{
Tomás Trewhela $\odot,,^{1, *}$ J. M. N. T. Gray $\odot,^{2}$ and Christophe Ancey ${ }^{1}{ }^{1}$ \\ ${ }^{1}$ Laboratory of Environmental Hydraulics, École Polytechnique Fédérale de Lausanne, Lausanne, Switzerland \\ ${ }^{2}$ Department of Mathematics and Manchester Centre for Nonlinear Dynamics, University of Manchester, \\ Oxford Road, Manchester M13 9PL, United Kingdom
}

(Received 20 November 2019; accepted 31 March 2021; published 21 May 2021)

\begin{abstract}
We studied the segregation of single large intruder particles in monodisperse granular materials. Experiments were carried out in a two-dimensional shear cell using different intruder and media diameters, whose quotient defined a size ratio $R$ that ranged from 1.2 to 3.333 . When sheared, the intruders segregated and rotated at different rates, which depended on their $R$ values and depth. The vertical intruder trajectories as a function of time were curved due to nonconstant depth-dependent segregation rates. An analysis that considered the lithostatic pressure distribution and a size ratio dependence was done to capture the trajectories and the general segregation rate behavior. As a result of a strain rate analysis, we observed a greater expansion rate around the intruders when $R$ values were larger, which in turn promoted faster segregation. Experiments with large $R$ values showed that intruder rotation was weak and local shear rates were low. In contrast, experiments with $R$ closer to unity resulted in strong intruder rotation, high local shear rates, and contraction below the intruder. Therefore, an intruder with a diameter close to that of the medium was likely to segregate due to a rotation mechanism. We propose that large particle segregation depends on size ratio, local expansion rate, and, to a lesser extent, the local shear rate. Based on our observations we redefine large particle segregation as two well-defined processes dependent on $R$ and the local strain rate.
\end{abstract}

DOI: 10.1103/PhysRevFluids.6.054302

\section{INTRODUCTION}

Polydisperse granular materials naturally segregate according to their species' size when sheared under gravity. Since $40 \%$ of all products use granular materials during their manufacture, particlesize segregation can be a major problem for industry, that often causes flow problems and degrades product quality [1,2], but can be very useful for sorting materials in agriculture and mining [3]. In natural environments, particle-size segregation can generate a range of complex phenomena, such as stratification patterns in avalanche deposits [4,5], flow fingering [6-8], static levees [9-11], front bulging [12,13], and self-channelization [14,15]. Segregation is therefore crucial in understanding the dynamics of geophysical mass flows $[16,17]$ and the dynamics of granular matter, in general [18].

A variety of particle segregation mechanisms have been identified and studied for various flow configurations $[19,20]$, with the segregation of large particles often called the Brazil nut effect in

\footnotetext{
*tomas.trewhela@epfl.ch
}

Published by the American Physical Society under the terms of the Creative Commons Attribution 4.0 International license. Further distribution of this work must maintain attribution to the author(s) and the published article's title, journal citation, and DOI. 
vibrated systems [21]. Simultaneously, the shear-induced mechanisms, (i) kinetic sieving [22,23] and (ii) squeeze expulsion, have been encountered widely in many granular flows, promoting their study [18]. Experimental observations and numerical simulations have described the mechanics of the kinetic sieving process precisely: it consists of small particles percolating through gaps generated by the relative movements of particle layers. The origin and nature of the squeeze expulsion mechanism, however, are not subject to a consensus. While it was defined originally by Savage and Lun [23] as imbalances in the contact forces applied on an individual particle which squeeze it out of its own layer into an adjacent one, other authors have proposed that the mechanism results from mass continuity or a net flux balance [24,25]. Therefore, a large particle will only rise if the surrounding voids are filled with percolating smaller particles. This assumption may hold for certain cases, but small particle percolation tends to be less pressure dependent and segregation fluxes have been found to be asymmetric [25-27]. This segregation flux asymmetry suggests that a connection between the two mechanisms may not be direct or independent of the particles' size ratio or the local particle concentration.

Efforts to explain why large particles segregate have been particularly intense in recent years. Guillard et al. [28] proposed a scaling of the forces acting on a large particle to define a segregation force. They found that this force was similar to a lift force and that it depended on the stress distribution. Despite this, Guillard et al. [28] did not address how a large intruder rises and how shear stress contributes to segregation. To address the question of why large particles segregate, van der Vaart et al. [29] proposed an analogy with the Saffman effect and introduced a buoyancylike force that depends on the size ratio. The origin of this granular Saffman effect is similar to viscous drag, but in their work this drag is exerted by a granular flow. Recently, Staron [30] failed to observe any liftlike force under flow conditions similar to those described by van der Vaart et al. [29]. Staron [30] concluded that force fluctuations around the intruder should be responsible for large particle segregation. Resistance is higher towards a rigid fixed bottom, hence any force imbalance pushes the intruder upwards. An analogy to a plunging object was proposed by Staron [30], based on previous work by Hill et al. [31], to illustrate the previous sentence. The role of interparticle friction and rotation in particle segregation was studied by Jing et al. [32] through numerical simulations. Jing et al. [32] found that large particle segregation was supressed when interparticle friction and rotation were negligible. They proposed that the rotation of a large particle is necessary for its segregation.

Particle-size segregation of a single large particle has been studied at the laboratory scale. van der Vaart et al. [27] considered large particles segregating in a simple shear cell, but their results focused on segregation flux asymmetry. Other studies measured lift and drag forces acting over intruders in granular media [33-35]. These intruders were held fixed or moved artificially, so no direct relation could be established between their results and the segregation of a single large particle. Recently, an experimental scaling for the segregation flux function was presented by Trewhela et al. [36]. In a three-dimensional shear box, similar to that of van der Vaart et al. [27], they found that the segregation rate of large particles was linear with the applied shear rate and the particles' size ratio.

Simple shear cells or boxes have been used previously to study granular and segregation processes (e.g., Refs. [27,36-38]). Stephens and Bridgwater [38] observed that the percolation rates and segregation mechanisms in simple shear cells were quite similar to those found in annular shear cells. These cells prescribe deformation so they impose a different flow configuration than those observed by Guillard et al. [28], van der Vaart et al. [29], and Staron [30].

We used a two-dimensional shear cell filled with small particles, in which one large particle (the intruder) was placed. In our experiments, shear was constant in depth but oscillated through time and the intruder moved freely towards the bulk free surface by the action of shear. Particle trajectories and velocity fields were determined using particle tracking velocimetry and interpolation, respectively. The strain rate tensor and its invariants were calculated to reveal how the granular material responded to external shear, as done in previous studies [35,39]. Various intruder and medium diameters were used to shed light on the role of size ratio in large particle segregation. 


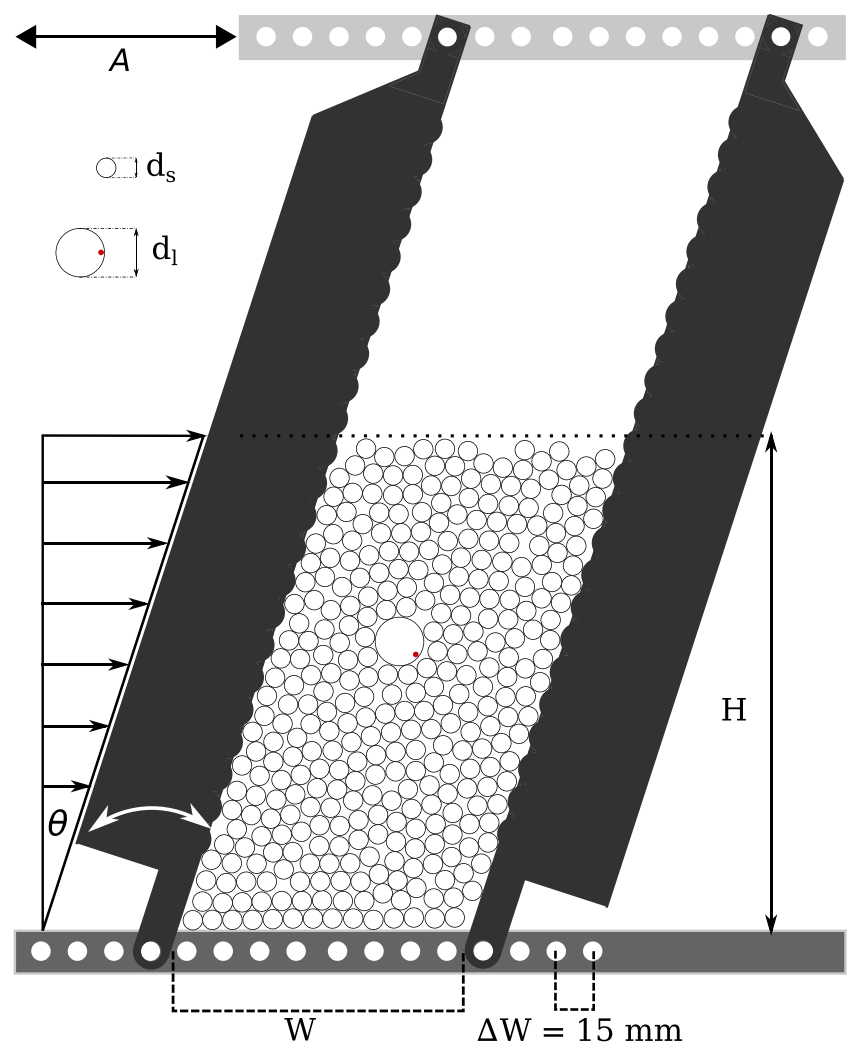

FIG. 1. Schematic diagram of the 2D shear cell setup, where $d_{s}$ is the diameter of the disks forming the surrounding granular medium, $d_{l}$ is the diameter of the intruder, and $A$ represents the amplitude generated by the cyclic movement of the plates. The bulk height $H=19 \mathrm{~cm}$ and the maximum plate inclination $\theta_{\max }=15^{\circ}$ were the same for all experiments. The cell width $W$ was changed for each $d_{s}$ to maintain a fixed ratio of $W / d_{s} \approx 14$ for all experiments.

\section{METHODS}

Experiments were carried out in a 5-mm-thick, two-dimensional (2D), shear cell consisting of two parallel polyvinyl chloride (PVC) side plates that rotated over axes located at their bases (see Fig. 1). The PVC side plates were corrugated and had a roughness that scaled to $d_{s}$. Cell width was set between $W=85$ and $145 \mathrm{~mm}$ in $\Delta W=15 \mathrm{~mm}$ steps. A granular material between the plates was sheared by their cyclic movements. Since the side plates were parallel, the externally imposed shear rate was independent of the depth but was periodic in time. The external shear rate is expressed by

$$
\dot{\gamma}_{e}(t)=\omega|\cos (\omega t)| \tan \theta_{\max },
$$

where $\theta_{\max }=15^{\circ}$ was the plates' maximum angle of inclination. The frequency $\omega=2 \pi / T$ was given by the period $T$, which varied slightly between experiments. To characterize and compare the shear rate exerted on average between experiments, we defined the mean shear rate as

$$
\dot{\gamma}_{m}=\frac{2 \omega}{\pi} \tan \theta_{\max }
$$

whose values for each experiment are presented in Table I. 
TABLE I. Mean shear rate $\dot{\gamma}_{m}$ in $\mathrm{s}^{-1}$ for each experiment using a $d_{s}=6,8$, and $10 \mathrm{~mm}$ media and a $d_{l}=10$, $12,14,18$, and $20 \mathrm{~mm}$ intruder.

\begin{tabular}{lccccc}
\hline \hline & & & & & \\
$d_{s}$ & 10 & 12 & 14 & 18 & 20 \\
\hline 6 & 0.052 & 0.056 & 0.055 & 0.057 & 0.055 \\
8 & 0.059 & 0.062 & 0.061 & 0.061 & 0.059 \\
10 & - & 0.064 & 0.056 & 0.053 & 0.064 \\
\hline \hline
\end{tabular}

A dry granular medium made of polyoxymethylene (POM) disks of diameter $d_{s}$ and an intruder disk of the same material, but of a different diameter $d_{l}>d_{s}$, were placed between the cell plates and glass panels. Three different disk diameters were employed as the surrounding media: $d_{s}=6,8$, and $10 \mathrm{~mm}$. Only disks larger than the medium's disk diameter were used as intruders: $d_{l}=10,12$, 14,18 , and $20 \mathrm{~mm}$. To quantify intruder rotation, a red dot was drawn on the edge of the intruder's circumference. POM has a density of $1.42 \mathrm{~g} \mathrm{~cm}^{-3}$ and a Young's modulus of $3000 \mathrm{MPa}$. According to Vaziri et al. [40], the frictional coefficient between POM cylinders sliding on each other at low velocities is 0.16 .

The single intruder was initially placed in the center of the cell at a height of $4 \mathrm{~cm}$, measured from the cell bottom to the lowest point of the intruder's circumference. The cell was then filled with the smaller disks up to a height of $19 \mathrm{~cm}$, creating an effective bulk height of $h=15 \mathrm{~cm}$ over the intruder. This latter condition was maintained for all experiments.

Due to the characteristics of the cell, the appearance of the Janssen effect [41] was an initial concern. However, initial experiments showed that intruder segregated faster towards the free surface. If the pressure were constant at depth, as the Janssen effect would suggest, there would be no physical quantity left to explain the variable segregation rate observed in our experiments. The Janssen effect is therefore, at worst, negligibly small.

\section{A. Image acquisition and particle tracking}

Experimental run times ranged from 15 to $70 \mathrm{~min}$. Each experiment was recorded using a Basler acA2000-165uc camera at four frames per second. The position and the radius of every POM disk were determined using a circular Hough-transform algorithm available in MATLAB. A particletracking algorithm was used to correlate positions to trajectories [42]. First, the intruder position $\boldsymbol{r}_{l}=\left(x_{l}, z_{l}\right)$ and its velocity $\boldsymbol{u}_{l}=\left(u_{l}, w_{l}\right)=\partial \boldsymbol{r}_{l} / \partial t$ were determined separately as functions of time $t$. Second, all particles' positions $\boldsymbol{r}_{m}$ and trajectories were used to calculate the particles' velocities $\boldsymbol{u}_{m}$. Finally, spatial interpolation of the particles' velocities at a certain time $t$ enabled the calculation of the entire bulk's velocity field $\boldsymbol{u}$.

\section{B. Trajectory analysis and segregation rate scaling}

A single large intruder of diameter $d_{l}$ segregating through a matrix of smaller particles of diameter $d_{s}$ can be analyzed considering that large particle concentration $\phi^{l}$ is almost 0 ; i.e., small particle concentration $\phi^{s}=1-\phi^{l}=1^{-}$. Such consideration is enough to consider that the intruder's vertical velocity $w_{l}=d z / d t$ is in fact equal to the segregation velocity magnitude $f_{s l}$ defined by Trewhela et al. [36] as

$$
f_{s l}=\mathcal{B} \frac{\rho_{*} g \dot{\gamma} \bar{d}^{2}}{\mathcal{C} \rho_{*} g \bar{d}+p} \mathcal{F}\left(R, 1^{-}\right),
$$

where $\mathcal{B}$ and $\mathcal{C}$ are empirically determined constants, $R=d_{l} / d_{s}$ is the particles' size ratio, $\rho_{*}$ is the particles' intrinsic density, $p$ is the pressure, $\bar{d}=d_{s} \phi^{s}+d_{l} \phi^{l}$ is the concentration-averaged particle diameter, and $\mathcal{F}$ is a function of $R$ and $\phi^{s}$. In the case of a single large particle surrounded by smaller 
particles, i.e., $\phi^{s}=1^{-}, \bar{d} \approx d_{s}$ and $\mathcal{F}\left(R, 1^{-}\right)=R-1$. We then simplify Eq. (3) by considering a lithostatic pressure distribution within the bulk, $p=\rho_{*} \Phi g(h-z)$, which results in the first-order differential equation

$$
\frac{d z}{d t}=f_{s l}=\mathcal{B} \frac{\dot{\gamma} d_{s}^{2}(R-1)}{\mathcal{C} d_{s}+\Phi(h-z)}
$$

where $\Phi$ is the solids volume fraction and $h$ is the bulk height as in our experimental setup. We solved Eq. (4) for the vertical position $z$ of the large intruder by using an initial condition $z=z_{0}$ at $t=0$ :

$$
\mathcal{Z}=\mathcal{C} d_{s}\left(z-z_{0}\right)+\frac{\Phi}{2}\left[\left(h-z_{0}\right)^{2}-(h-z)^{2}\right]=\mathcal{B} \dot{\gamma} d_{s}^{2}(R-1) t=\mathcal{K} t,
$$

where the variable $\mathcal{Z}$ represents a parametrized trajectory. For each experiment, a different constant $\mathcal{K}$ can be determined by fitting the explicit theoretical trajectory,

$$
z(t)=\frac{1}{\Phi}\left[\mathcal{C} d_{s}+\Phi h-\sqrt{\mathcal{C}^{2} d_{s}^{2}+2 \mathcal{C} d_{s} \Phi\left(h-z_{0}\right)+\Phi^{2}\left(h-z_{0}\right)^{2}-2 \Phi \mathcal{K} t}\right],
$$

to the experimental trajectory of the intruder. The empirical constants $\mathcal{B}$ and $\mathcal{C}$ can be determined using a least-squares fit to the entire experimental data. In the work of Trewhela et al. [36] these constants were found to be $\mathcal{B}=0.374$ and $\mathcal{C}=0.271$ for a three-dimensional granular bulk of borosilicate glass beads submersed in a refractive-index-matched fluid mixture of ethanol and benzyl alcohol.

\section{Intruder rotation}

Red dot identification and tracking were done simultaneously to intruder tracking. The dot's position $\boldsymbol{r}_{d}$ and movement, relative to the intruder's position, were used to estimate the intruder's angular velocity $\boldsymbol{\Omega}_{l}=4 \boldsymbol{r}_{d} \times \boldsymbol{u}_{d} / d_{l}^{2}$. Since rotation had no preferential direction, we were interested in the magnitude of $\boldsymbol{\Omega}_{l}$, so its norm was considered as relevant $\Omega_{l}=\left|\boldsymbol{\Omega}_{l}\right|$.

A conditional probability $P\left(w_{l} \mid \Omega_{l}\right)=P\left(w_{l}, \Omega_{l}\right) / P\left(\Omega_{l}\right)$ was calculated to quantify the occurrence of segregation and rotation. This probability was determined from a bivariate probability distribution function (pdf) of the time series of the intruder's vertical velocity $w_{l}$ and angular velocity $\Omega_{l}$. The bivariate pdf $P\left(w_{l}, \Omega_{l}\right)$ was calculated using MATLAB's mvnpdf function. The marginal probability distribution function was determined using MATLAB's pdf function.

\section{Strain rate tensor invariants}

The strain rate tensor $\boldsymbol{D}=\frac{1}{2}\left(\nabla \boldsymbol{u}+\nabla \boldsymbol{u}^{\prime}\right)$ was estimated from the velocity field $\boldsymbol{u}$. The first invariant of the strain-rate tensor is called the expansion rate,

$$
I_{D}=\operatorname{tr}(D)=\nabla \cdot u
$$

and the second invariant is defined as

$$
I I_{D}=\left(\frac{1}{2} \operatorname{tr}\left(S^{2}\right)\right)^{1 / 2}
$$

where $S=-\frac{1}{2} I_{\boldsymbol{D}} \mathbf{1}+\boldsymbol{D}$ is the deviatoric strain rate tensor. This second invariant is half the shear rate $\dot{\gamma}=2 I I_{\mathrm{D}}$ [43]. Both invariants were estimated from the velocity fields, which themselves resulted in the fields $I_{D}(x, z)$ and $I I_{D}(x, z)$ for each time step.

To analyze the local strain rate around the intruder, we evaluated $I_{D}$ and $I I_{D}$ on the intruder's circumference. Based on the intruder's position and diameter, we split the intruder circumference into arcs. We evaluated and extracted each invariant value from the middle arc points $\phi_{l}$. This method allowed us to evaluate both strain-rate tensor invariants around the intruder: $I_{\boldsymbol{D}_{l}}\left(\phi_{l}\right)$ and $I I_{D_{l}}\left(\phi_{l}\right)$. 

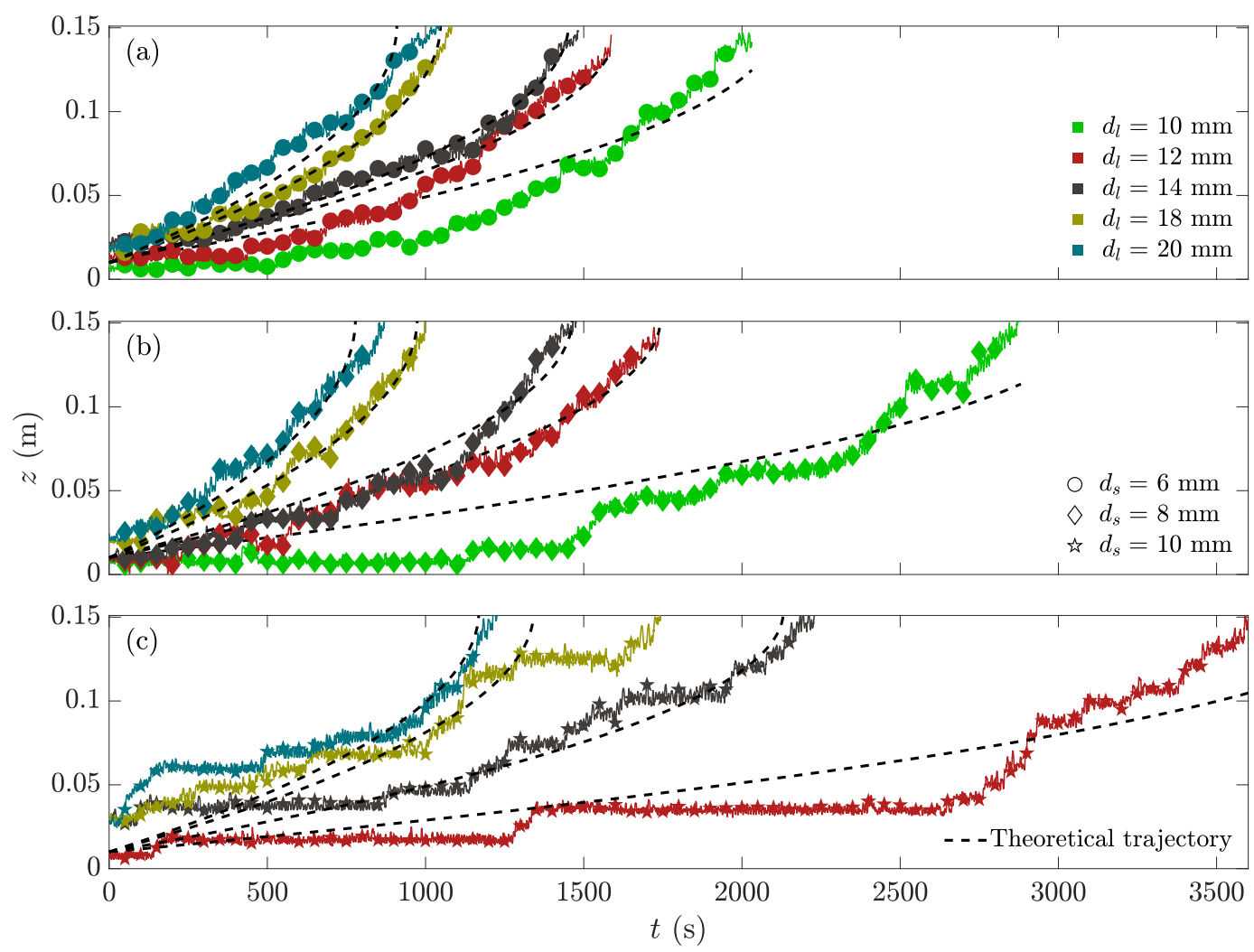

FIG. 2. Vertical position $z$ as a function of time $t$ for the intruders of $d_{l}=10,12,1418$, and $20 \mathrm{~mm}$ [in colors, see legend in panel (a)] segregating through the (a) $d_{s}=6 \mathrm{~mm}(\bullet)$, (b) $8 \mathrm{~mm}(\bullet)$, and (c) $10 \mathrm{~mm}(\star)$ medium. The dashed lines plot the theoretical fits [see Eq. (6)] to the experimental trajectories, all of which used a value of $\mathcal{C}=0.271$ and $\Phi=0.7$.

\section{RESULTS}

\section{A. Vertical position}

The intruder's vertical position (see Fig. 2) and the bulk's velocity field were the first results obtained from the images. Near the bottom, at the beginning of the experiment, segregation was considerably slower than in the upper regions. The closer the intruder got to the free surface, the faster it moved. The intruder generally showed a nonlinear, depth-dependent segregation rate in all the experiments.

For all our results we used a size ratio definition $R=d_{l} / d_{s}$, the intruder diameter divided by the media diameter as considered by Trewhela et al. [36]. As shown in Fig. 2, the segregation rate of the large particle surrounded by the 6-mm disks increased proportionally with $R$. These findings also held for experiments using larger medium diameters $d_{s}=8$ and $10 \mathrm{~mm}$ [see Figs. 2(b) and 2(c)]. A laddering, almost stepwise ascent, was observed in these cases, especially in the $d_{s}=10 \mathrm{~mm}$ medium experiments [ $\star$ in Fig. 2(c)].

All the intruders demonstrated oscillatory vertical movement. Indeed, due to the plates' cyclic movement, the intruders moved upwards and downwards when the bulk was sheared. This movement could be interpreted as noise relative to an average vertical position during a cycle. Cyclical vertical movement was observed throughout the entire experiment and exhibited the same amplitude, independent of $z$. The magnitude of this movement did not change between experiments, even when different intruder diameters were used, as shown in Fig. 2. It is important to note that the bulk 


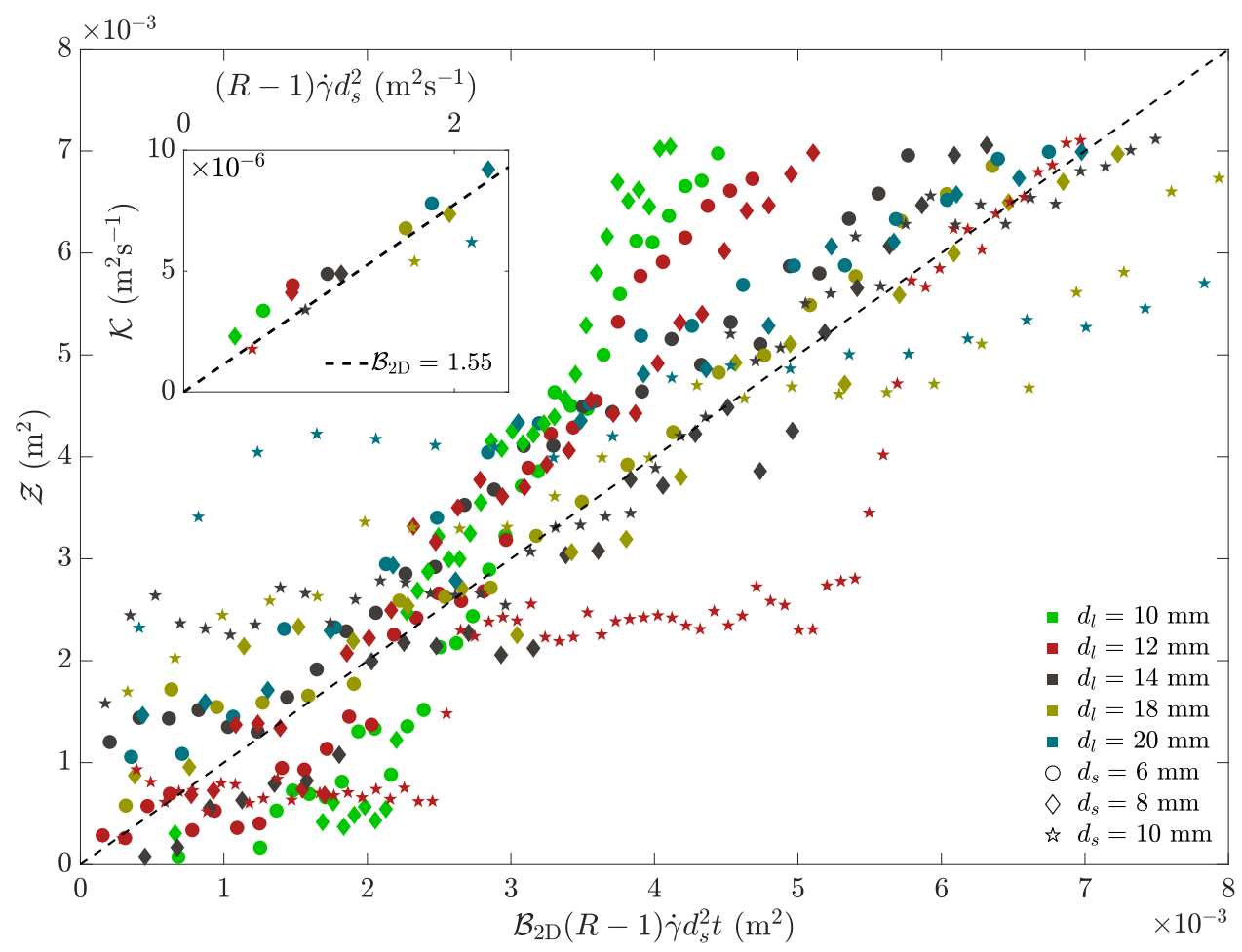

FIG. 3. All the intruder trajectories collapsed onto the identity dashed line given by the parametrized depth $\mathcal{Z}$ and time $\mathcal{B}_{2 \mathrm{D}}(R-1) \dot{\gamma} d_{s}^{2} t$. The inset plot shows the fitted constants $\mathcal{K}$ for each experiment as a function of $(R-1) \dot{\gamma} d_{s}^{2}$. The dashed line in the inset plot has a slope of $\mathcal{B}_{2 \mathrm{D}}=1.55$ and it is determined by a linear regression of $\mathcal{K}$ as a function of $(R-1) \dot{\gamma} d_{s}^{2}$.

media were sheared cyclically, so the oscillatory vertical movement was a result of the setup and not due to the segregation process.

We fitted the theoretical trajectory presented in Eq. (6) to each of the experimental trajectories of the intruder. These theoretical trajectories are represented by dashed lines in Fig. 2. The experimental and the theoretical trajectories were in good agreement using the framework proposed in Sec. II B. The determination coefficient $r^{2}$ for the fits ranged from 0.74 to 0.98 , with no particular dependence on to $R, d_{l}$, or $d_{s}$. The fits were done via least squares and used a value for $\mathcal{C}=0.271$ following the results of Trewhela et al. [36]. A value of $\Phi=0.7$ was used for the analysis and was a result of averaged calculations done for the whole media. The role of the $\mathcal{C}$ constant is to provide a finite gradient for the curves when the intruder arrives to the surface, which is particularly helpful for numerical methods. The variability of the quadratic fit does not change much, if $\mathcal{C}$ is set to zero, a fact also pointed out by Trewhela et al. [36]. Therefore, for each experiment we obtained a fitted parameter or a constant $\mathcal{K}$ which is representative of the segregation rate of that experiment and that does not change much with the value of $\mathcal{C}$.

In agreement with the presented framework in Sec. II B, the $\mathcal{K}$ constant is a function of the $R, \dot{\gamma}$, and $d_{s}$ parameters. In the inset of Fig. 3 we plotted the determined $\mathcal{K}$ constants, defined in Eq. (5), as a function of the experimental parameters. A clear linear relation is observed between $\mathcal{K}$ and those parameters, and a linear regression of the data was done to obtain the slope of such linear function. An empirical constant $\mathcal{B}=\mathcal{B}_{2 \mathrm{D}}=1.55$ was determined for our experimental dataset. This is different from the value of $\mathcal{B}=\mathcal{B}_{3 \mathrm{D}}^{\text {wet }}=0.374$ determined by Trewhela et al. [36] in their fluid saturated three-dimensional shear box setup and from the buoyancy corrected value of $\mathcal{B}=\mathcal{B}_{3 \mathrm{D}}^{\text {dry }}=0.7125$ 
for an equivalent dry system. The approximately factor of 2 difference between $\mathcal{B}_{2 \mathrm{D}}$ and $\mathcal{B}_{3 \mathrm{D}}^{\text {dry }}$ may be due to the two-dimensional rather than three-dimensional flow configuration. The determination coefficient for $\mathcal{B}_{2 \mathrm{D}}$ was $r^{2}=0.87$. We also repeated the method done by Trewhela et al. [36] to calculate $\mathcal{B}$ using a least-squares algorithm on the whole experimental dataset. We determined a value of $\mathcal{B}_{2 \mathrm{D}}=1.457$, which was still different from the dry value found by Trewhela et al. [36], but was slightly different from the value determined with the linear regression analysis of the $\mathcal{K}$ constants.

The empirical constant $\mathcal{B}_{2 \mathrm{D}}$ for our dataset was used to collapse all of our experimental data onto a single identity line. Figure 3 shows all the intruder's vertical trajectories parametrized under the variable $\mathcal{Z}$ as a function of the parametrized time $\mathcal{B}_{2 \mathrm{D}}(R-1) \dot{\gamma} d_{s}^{2} t$. Despite some general disagreements, most likely due to particle diffusion, the trajectories collapse well under the proposed scaling. We see in Fig. 3 that the experiments with the most disagreement with the proposed scaling are the experiments carried out with $d_{s}=10 \mathrm{~mm}$ ( $\star$ in Fig. 3). This result can be explained if we consider that particle diffusion $\mathcal{D}$ scales to $\dot{\gamma} d_{s}^{2}$ [44]. Then, diffusion is considerably larger for $d_{s}=10 \mathrm{~mm}$ experiments than for $d_{s}=6$ or $8 \mathrm{~mm}$ experiments. Diffusion may be sufficient to explain these differences, but other effects like nonstrictly constant $\Phi$ or particle discretization, most visible in large $d_{s}$ experiments, could also be affecting these results.

Because no kinetic sieving mechanism was observed using the 2D shear cell configuration, we do not show any results on the percolation of small intruders through granular media made of large disks. We observed that when a single smaller intruder was introduced into the cell, it did not percolate down through the bulk. Small disks moved erratically on top of the upper layer until they found lateral gaps generated by the plate roughness, which we considered biased.

We did not observe a plateau for the segregation rate as a function of $R$, measured via the parameter $\mathcal{K}$ (see inset plot of Fig. 3). A constant value for $\mathcal{K}$, independent of the $R$ value, would have indicated that a maximum segregation rate can be achieved at a certain $R$ threshold. Although some authors pointed out that maximum segregation rates were achieved at $R$ values of 2 [26], 1.7 [45] or 2.5 [46], it was not the case for our two-dimensional shear cell experiments in the range of $R=[1.2,3.33]$. This discrepancy to previous studies is due to the shear cell configuration that prescribes the shear rate and maintains a relatively constant solid volume fraction. In other experimental or numerical setups, the flow is left to evolve freely, and shear rate, pressure, or the solid volume fraction enter into a highly nonlinear feedback with the flow.

\section{B. Intruder rotation}

Intruder rotation was observed as the bulk was sheared during each cycle. In some experiments the intruder rotated more, especially when intruder sizes were close to those of the media. Rotational movement did not tend towards any particular direction, and it was not necessarily synchronized with plate movement. Notably, in some cases we observed that the upwards movement of the intruder occurred simultaneously with its rotation.

Dot positions relative to the intruder's position are shown in Fig. 4. The red dot on the intruder's circumference is plotted relative to the intruder's position. Figure 4 shows that intruder rotation was highest for size ratios close to 1 . For example, the $d_{l}=12 \mathrm{~mm}$ intruder surrounded by $d_{s}=10 \mathrm{~mm}$ disks rotated around its center several times, which was reflected by the fact that the red dot's trajectory drew a complete circumference (Fig. 4, bottom row, left-hand panel). Whereas intruders with a small $R$ value completed several revolutions, intruders with large $R$ sometimes could not even complete one. This fact is shown in the top row, far-right panel of Fig. 4, where the $d_{l}=20 \mathrm{~mm}$ intruder surrounded by $d_{s}=6 \mathrm{~mm}$ disks barely rotated. In this case the red dot was never oriented downwards or to the left of the intruder's center (Fig. 4, top row, far-right panel). Experiments using the $d_{s}=8 \mathrm{~mm}$ media showed intermediate results, but still highly related to the corresponding $R$ values. Similar $R$ values exhibited similar results; the intruders' dots covered similar portions of the intruders' perimeter (see $R=1.8$ and 2 in Fig. 4) which points out that intruder rotation depends solely on $R$ and not on $d_{s}$ or $d_{l}$ particularly. Since segregation rates were also $R$ dependent, these 

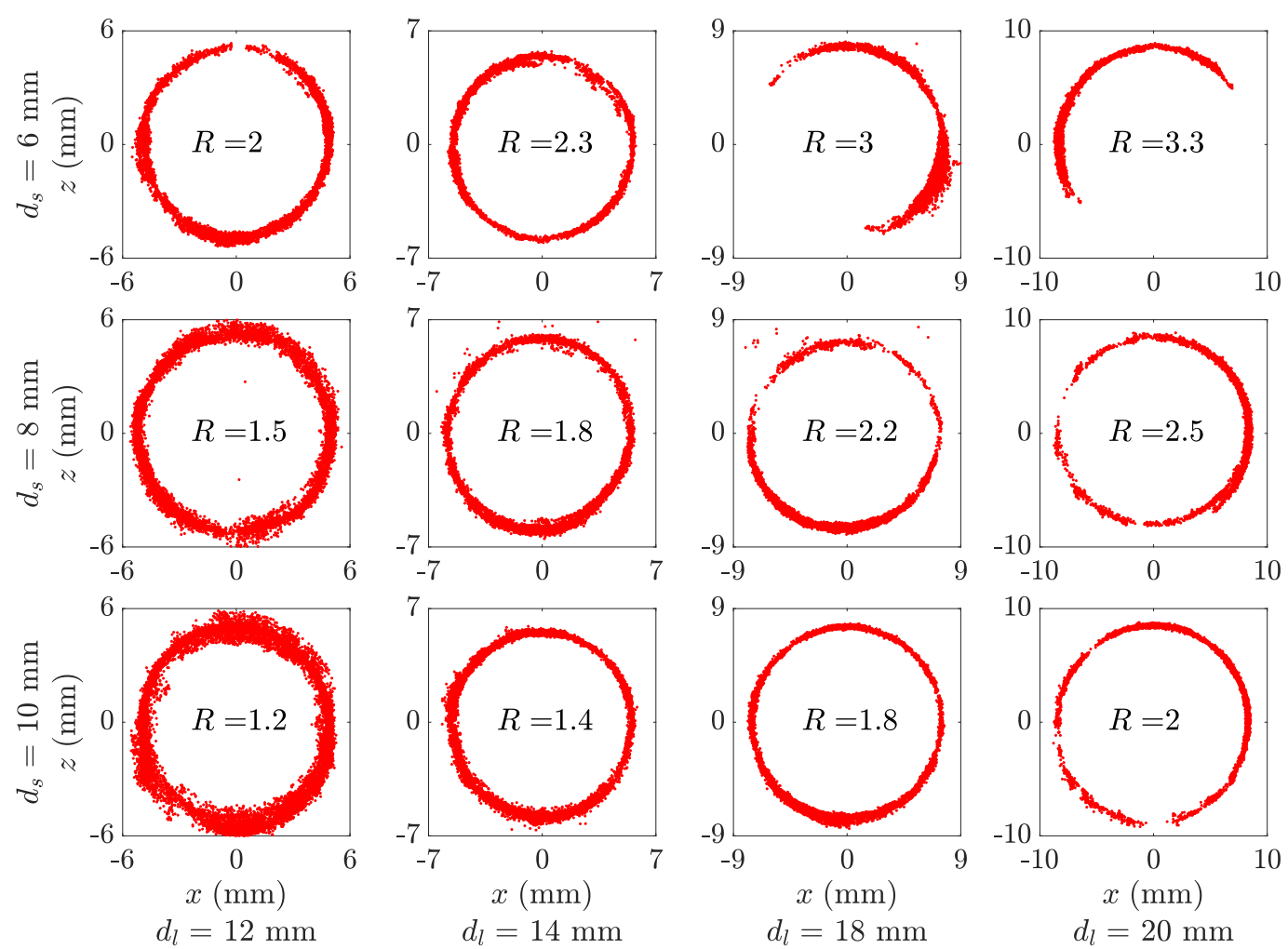

FIG. 4. Dot positions relative to the intruder's position. Top row (left to right): Experiments using a $d_{s}=$ $6 \mathrm{~mm}$ medium, with $d_{l}=12,14,18$, and $20 \mathrm{~mm}$ intruders. Middle row (left to right): Experiments using a $d_{s}=8 \mathrm{~mm}$ medium, with $d_{l}=12,14,18$, and $20 \mathrm{~mm}$ intruders. Bottom row (left to right): Experiments using a $d_{s}=10 \mathrm{~mm}$ medium, with $d_{l}=12,14,18$, and $20 \mathrm{~mm}$ intruders.

results indicate that there might be a relation between large particle segregation and large particle rotation for small $R$ values, lower than 2 and closer to 1 . Conversely, the lower rotation activity and high segregation rate observed in experiments with $R>2$ suggest that intruders segregate differently, and the segregation mechanism depends on $R$ as well.

Results plotted in Fig. 4 are for the whole run time of each experiment, a duration that was quite different and dependent on the size of the intruder as seen in Sec. III A. For shorter time intervals, for example, the duration of the shortest experiment, this trend is still preserved. Smaller intruders rotated more than larger intruders during equal time intervals.

Figure 5 shows that $\Omega_{l}$ was slightly correlated to vertical velocity $w_{l}=d z / d t$, which approximated to the segregation rate. Another interesting feature was the increasing values of $\Omega_{l}$ as intruders rose to the surface. This increment was especially relevant for size ratios $R<2.5$ as seen in Fig. 5, where we saw higher magnitudes for $\Omega_{l}$ and a tendency for even higher $\Omega_{l}$ values as the intruder approached the free surface. We suspect that the higher $\Omega_{l}$ values reached at the end of the experiment were a consequence of a combined lower pressure and lower solid's volume fraction close to the free surface.

To illustrate the link between rotation and segregation, the right column of Fig. 5 shows their conditional probabilities $P\left(w_{l} \mid \Omega_{l}\right)$. As detailed in Sec. II C, $P\left(w_{l} \mid \Omega_{l}\right)$ expresses the probability that the intruder moved vertically upwards given that it rotated (Fig. 5, right column). Experiments with $R<2.5$ indicate higher probabilities that the intruder segregated given that it had rotated. Conversely, when $R>2.5$, probabilities that the intruder segregated given that it had rotated were 

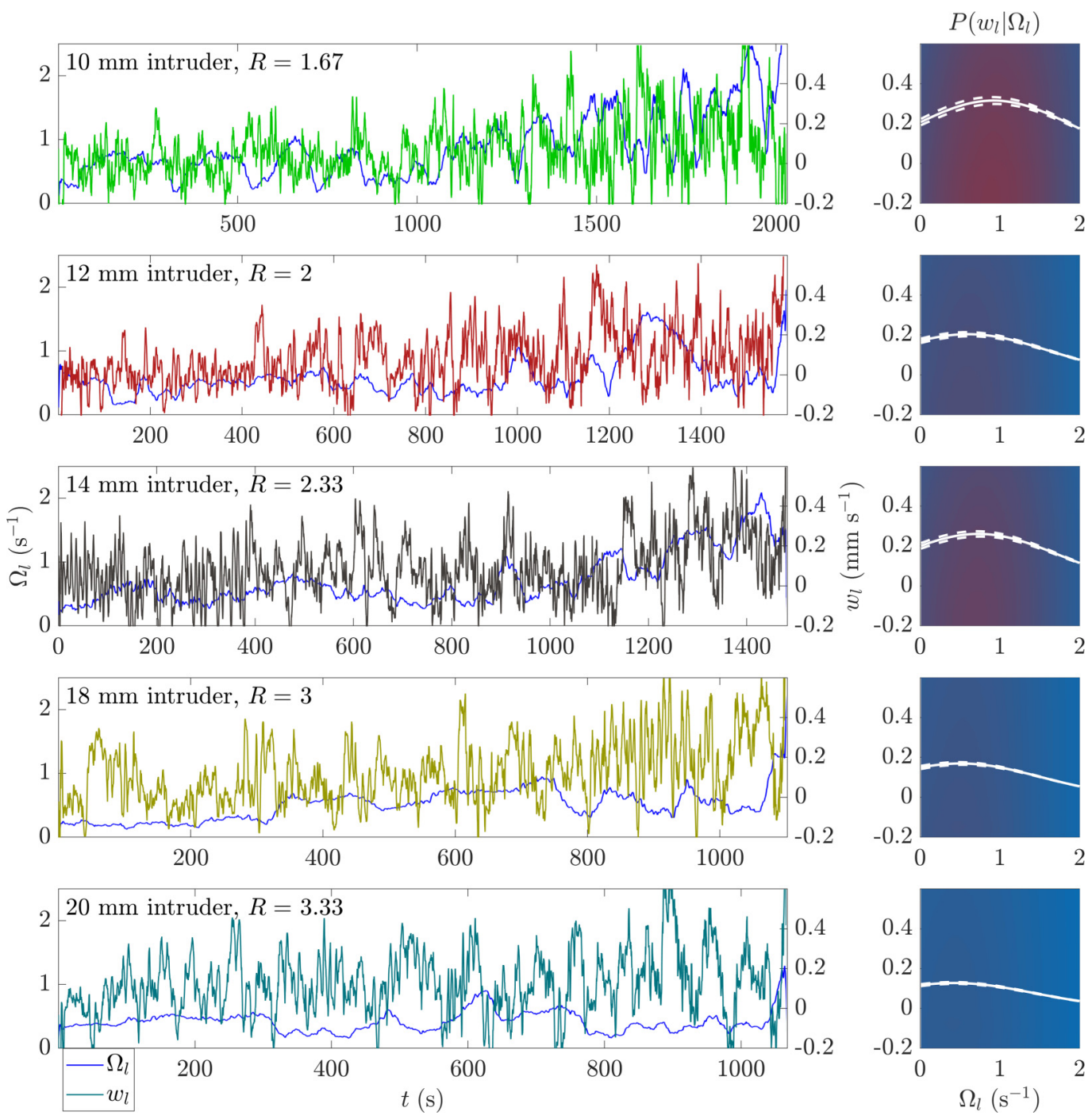

FIG. 5. Left column: Magnitude of the intruders' angular velocity $\Omega_{l}$ (left axis, blue line) and vertical velocities $w_{l}$ (right axis, different colors) as a function of time $t$ for experiments using the $d_{s}=6 \mathrm{~mm}$ medium and intruders of diameters $d_{l}=10,12,14,18$, and $20 \mathrm{~mm}$ (size ratios $d_{l} / d_{s}=1.67,2,2.33,3$, and 3.33). Right column: Probability of $w_{l}$ given that $\Omega_{l}, P\left(w_{l} \mid \Omega_{l}\right)$. Red tones indicate a higher probability, with a maximum value of 0.5 , and blue tones indicate a lower probability, with minimum value of 0 . The continuous white line draws the mean values and the dashed white lines draw the mean values plus and minus standard deviations.

lower. For each run, the probabilities of having a certain $\Omega_{l}$ value were averaged and plotted (Fig. 5, white lines over colormaps). These averages and deviations were calculated to highlight the magnitude differences between runs with different size ratios. These results confirm that as the size ratio $R$ increased, intruders had lower probabilities of segregating given that they had rotated, and their rotation was weaker than that observed for size ratios values that were closer to 1.

Figure 5 shows that, in general, $\Omega_{l}$ showed greater variability for $R<2$ experiments. The experiment with $R=1.67$ displayed the highest mean values for rotation, with a maximum at $\Omega_{l} \sim 2.5 \mathrm{~s}^{-1}$. For the rest of the experiments, their maximum values for $\Omega_{l}$ decreased as $R$ increased, as well as their conditional probabilities $P\left(w_{l} \mid \Omega_{l}\right)$. 

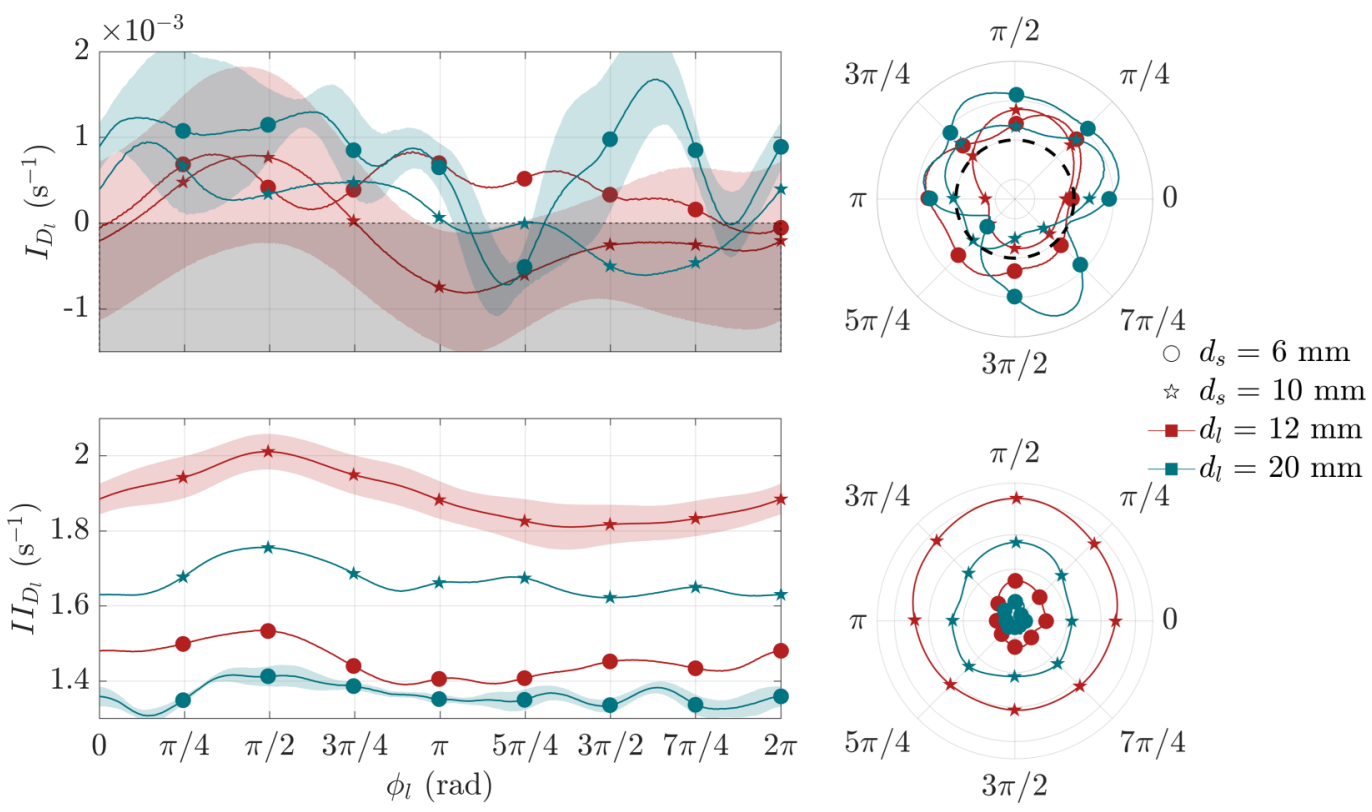

FIG. 6. Left column: Time-averaged strain rate tensor invariants $I_{\boldsymbol{D}_{l}}$ (top row) and $I I_{\boldsymbol{D}_{l}}$ (bottom row), around the intruder's circumference $\phi_{l}$, with the angle measured counterclockwise from the horizontal direction towards the right of the cell (3 o'clock). Colored areas represent values and their standard deviations. The gray area represents contraction. Right column: Polar plots of the same strain rate tensor invariants for the experiments with $d_{s}=6(\bullet)$ and $10(\star) \mathrm{mm}$ media, and $d_{l}=12$ (red) and 20 (turquoise) intruders. Standard deviations were not plotted for all experiments for visualization purposes.

\section{Strain rate tensor invariants}

Figure 6 shows the strain rate tensor invariants around the intruder's circumference, $I_{D_{l}}$ and $I I_{D_{l}}$, using both Cartesian and polar coordinates (Fig. 6, left and right columns, respectively). In the polar coordinates plot, the angle $\phi_{l}$ was measured counterclockwise from the horizontal direction towards the right of the cell (3 o'clock). To represent the experimental results of $I_{D_{l}}\left(\phi_{l}\right)$ and $I I_{D_{l}}\left(\phi_{l}\right)$, we took their time-averaged values over the entire experiment. In general, the mean values for both invariants depended on the size ratio. A second general observation was that $I_{\boldsymbol{D}_{l}}$ and $I I_{\boldsymbol{D}_{l}}$ were greatest on the upper half of the intruder's circumference, in accordance with the observed upward movement. The majority of the experiments showed maximum values at $\phi_{l}=\pi / 2$ and minimum values at $\phi_{l}=3 \pi / 2$ for both invariants. On average, greater values are found on the upper half of the intruder and smaller values are found on its lower half. These results showed that the intruder moved towards regions where $I_{D_{l}}$ and $I I_{D_{l}}$ were greater, thus to the free surface.

$I_{D_{l}}$ tended to be positive between 0 and $\pi$ and negative elsewhere (contraction). For the experiment with $R=1.2$ (red $\star$ in Fig. 6), the arc where $I_{D_{l}}>0$ is particularly narrow (between $\pi / 8$ and $3 \pi / 4)$. This result suggests that for size ratios close to 1 , gap formation was limited due to weak size heterogeneity. On the contrary, for $R=3.33$ (turquoise $\bullet$ in Fig. 6), $I_{\boldsymbol{D}_{l}}$ is positive almost anywhere around the intruder's circumference. Grain movement creates microscopic expansion and segregation is enhanced. This grain movement resulted in faster intruder segregation, as shown in the inset of Fig. 3. The contraction measured below the intruder, with small particles tightly filling the gaps beneath it, explains why large particles had difficulties in moving to the cell's bottom.

Local shear rate values for each experiment depended on $d_{s} / d_{l}$ as well. The values of $I I_{D_{l}}$ were always positive by definition, with its highest values observed between 0 and $\pi$ and its local maximum also at $\pi / 2$. Surprisingly, size ratios close to 1 showed higher $I I_{\boldsymbol{D}_{l}}$ values. However, this observation was consistent with the argument that rotation and angular velocity play a role in the 
segregation of large particles. Shear rate is related to angular deformation, which was observed experimentally by intruder rotation. The magnitudes of $I I_{D_{l}}$ are of the same order of magnitude as the average external shear rate $\dot{\gamma}_{m}=2.67 \times 10^{-2} \mathrm{~s}^{-1}$ (Table I). Even though all the experiments shared the same externally imposed shear rate, $I I_{\boldsymbol{D}_{l}}$ was locally distributed around the intruder's circumference at values ranging between approximately $1.8 \times 10^{-2}$ and $2 \times 10^{-2} \mathrm{~s}^{-1}$ (Fig. 6). Also, the mean values of $I I_{D_{l}}$ around the intruder's circumference are dependent on the size ratio. These mean values show differences of $6 \times 10^{-3} \mathrm{~s}^{-1}$ between the experiments with size ratios of 1.2 and 3.33 (see Fig. 6, red $\star$ and turquoise $\bullet$, respectively).

Figure 6 also presents two intermediate cases with $R=2$ for particle diameters of 6 and $10 \mathrm{~mm}$ and intruders of 12 and $20 \mathrm{~mm}$, respectively. Even though the size ratios are the same, the values calculated for $I_{\boldsymbol{D}_{l}}$ and $I I_{\boldsymbol{D}_{l}}$ are different, with mean differences of $5 \times 10^{-4}$ and $2 \times 10^{-4} \mathrm{~s}^{-1}$, respectively. We think these differences are due to the plate roughness and slightly different $W / d_{s}$ values.

\section{Segregation mechanism}

In their description of the squeeze expulsion mechanism, Savage and Lun [23] provided no clear role for the particles' size ratio. Our results in Sec. III suggest that segregation is caused by a combination of local expansion rate and rotation that depends on the size ratio $R=d_{l} / d_{s}$. High microscopic values for $I_{D_{l}}$ were observed for experiments with large $R$ values and segregation rates were greater in those cases. $\boldsymbol{I}_{\boldsymbol{D}_{l}}$ faded as $R$ decreased, but segregation still happened. For $R$ tending to 1 , rotation and $I I_{D_{l}}$ became predominant, and they were significant for segregation. However, for $R>2$, segregation rates were considerably higher; thus, local expansion rate was a much more effective submechanism for segregation than rotation was. Nonetheless, the rotation's contribution for relatively smaller intruders is still key for their segregation.

Based on our experiments, two processes occur in an initially dense granular material that undergoes shear [see Figs. 7(a) and 7(e)]:

(i) If $I_{D}$ around the intruder is large enough, small particles entrain beneath the large intruder. This small-particle entrainment may lift the intruder up, presumably through the normal stress redistribution. This occurrence of entrainment does not depend solely on microscopic expansion rate increments. All our experiments were subjected to very similar macroscopic shear rates $\dot{\gamma}_{m}$ (Table I) and effective bulk height $h$, yet segregation rates differed (see inset of Fig. 3). Therefore, the second variable controlling the entrainment should be $R$. When $R>2$ it becomes easier for disks surrounding the intruder to entrain. For $R$ values close to unity, entrainment is less frequent, due to weak gap generation, and the intruder usually remains in its place.

(ii) Shear-induced local expansion redistributes forces around the intruder. As a result, the intruder may become interlocked with its neighbors. Normal stresses transmitted through the intruder's neighbors create a force network that restrains the intruder's movement. When shear continues to be applied, the interlocked particles move conjointly around a pivot below them. Similarly to the first process, this rotational movement depends on $R$. Our results indicated higher rotation, a greater probability $P\left(w_{l} \mid \Omega_{l}\right)$, and higher local shear rates $I I_{D_{l}}$ for $R<2$ (Fig. 5). A size ratio close to 1 indicates that interlocking is likely to be occurring. It is plausible that slight size differences between the intruder and the medium require fewer surrounding particles to lock-in the intruder. However, our experiments showed that the probability of interlocking remains low. Therefore, the segregation caused by this process is slower and less effective than that caused by the first process.

\section{CONCLUSIONS}

A two-dimensional, oscillatory shear cell was used to study the segregation of a large particle intruder through a medium of smaller particles. The intruder's position and rotation were measured 


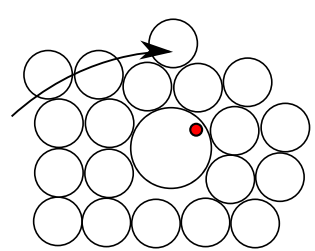

(a)

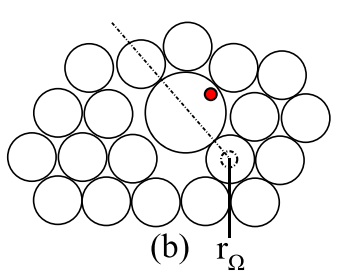

(b) $\mathrm{r}_{\Omega}$

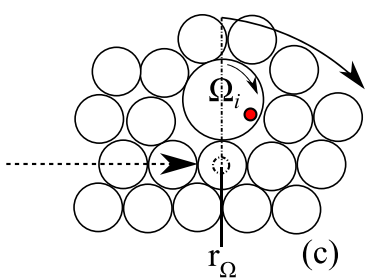

(c)

(d)
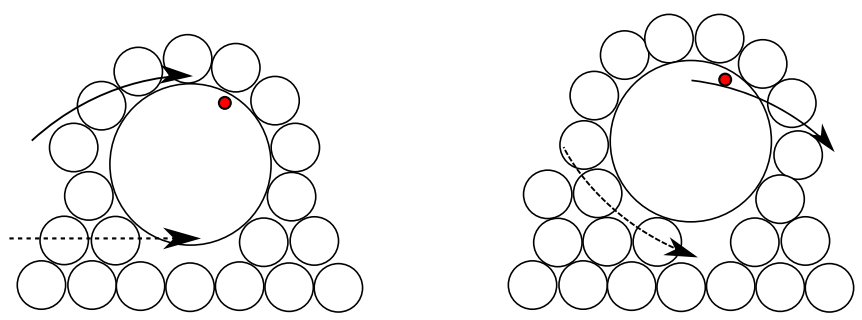

(e)

FIG. 7. Schematic diagram of the segregation of a single large intruder of $d_{l}=10$ [top row: (a), (b), and (c) Rotation-based mechanism] and $20 \mathrm{~mm}$ [bottom row: (d) and (e) Expansion-based mechanism] under the action of an external shear rate, $\dot{\gamma}_{m}$. (a) Surrounding particles lock the intruder, which form a (b) stress axis $r_{\Omega}$ that passes through the intruder, and (c) further shear rotates the axis around a base pivot point, and hence the lock intruder rotates on top of the pivot point, segregating. (d) The granular bulk locally dilates around the intruder creating gaps for (e) surrounding particles to entrain beneath the intruder, lifting the intruder and segregating it. In the Appendix, we present images showing these mechanisms, and videos showing these mechanisms are provided as Supplemental Material [47].

and tracked over time. We found that the segregation rate was a nonlinear function of time, dependent on the intruder's depth and the size ratio $R=d_{l} / d_{s}$ between the intruder and the medium diameter. In a fashion similar to that of the results found by Trewhela et al. [36], we fitted quadratic curves to the experimental trajectories based on a lithostatic pressure distribution. With this assumption, we validated the scaling of Trewhela et al. [36] for a two-dimensional configuration and in particular the observation that an increase in $R$ increased proportionally the segregation rate. Intruder rotation, quantified in terms of angular velocity, was found to be more frequent and intense, the lower and closer to $1 R$ was. We conclude that intruder rotation is a relevant mechanism in the segregation of large particles at small size ratios, in agreement with the proposition of Jing et al. [32].

Using a different setup and flow configuration, we found the same segregation behavior as that presented by several authors $[26,28,48]$, large particles segregated, predominantly, towards regions where the microscopic expansion rate was greater. Complementarily, we found that for size ratios close to 1 shear rate becomes a relevant variable for segregation. Based on our observations, particles subjected to high microscopic $I I_{D}$ values rotate more, which facilitates their segregation despite low microscopic values of $I_{D}$. However, a high local $I I_{D}$ value is not as predominant as $I_{D}$ for a fast segregation rate. Intruders subjected to high microscopic expansion rates segregated faster. Even though we did not present stress measurements, we presented a plausible explanation for the role of the local shear-stress gradient in the segregation of large particles.

Based on the observations presented here, we have suggested a detailed description of the squeeze expulsion mechanism through two distinguishable processes. The first process is strongly dependent on microscopic expansion, whereas the second depends on rotation, i.e., governed by the local shear rate. Frustration of the rotation-based process depends on surrounding interparticle contacts, which was observed for $R>2$ where the intruder needed more particles in close contact to interlock. We 


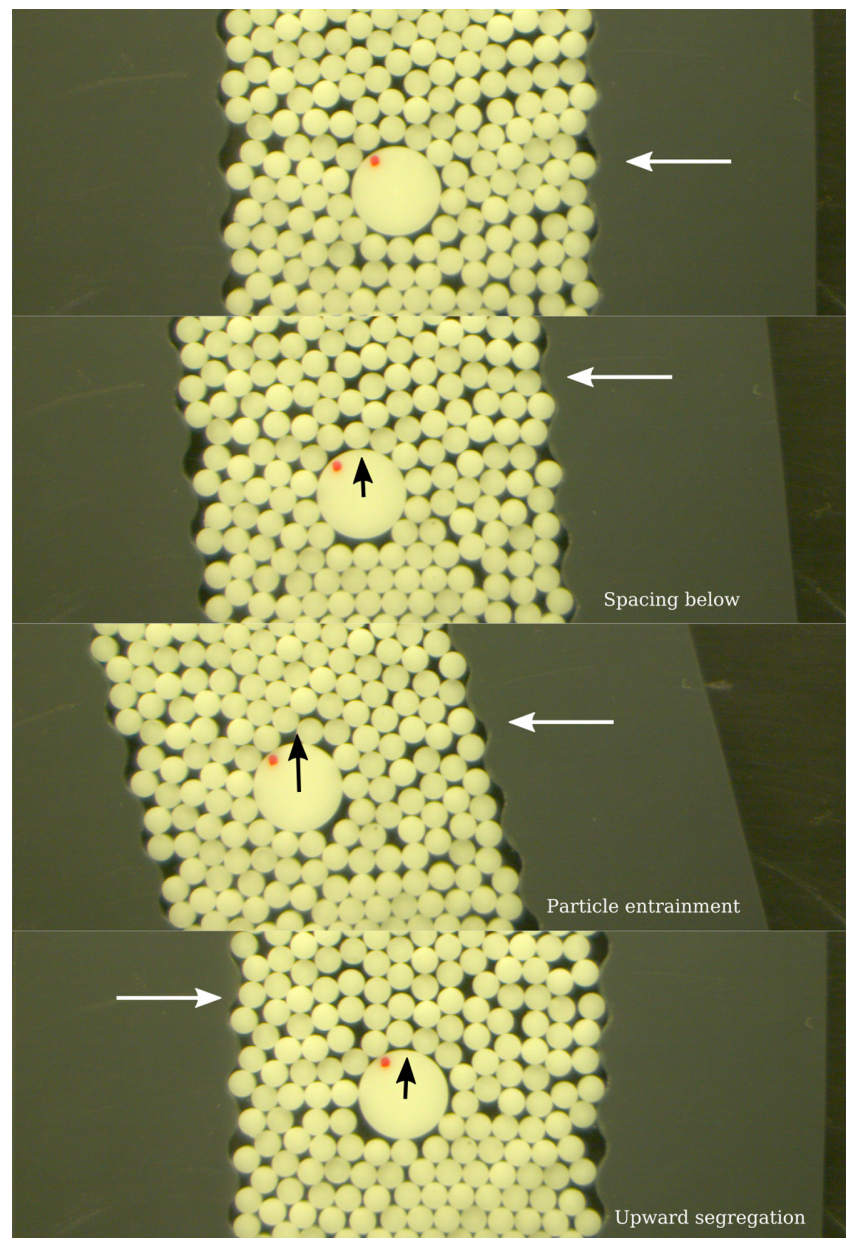

FIG. 8. Image sequence of the expansion-dominant segregation mechanism. A 20-mm intruder segregates upwards due to the squeezing action exerted by the surrounding 6-mm particles, which entrain underneath the intruder.

proposed that the occurrence of these processes, although independent of each other, are highly dependent on the particles' size ratio $R$.

\section{ACKNOWLEDGMENTS}

The authors acknowledge the support of the Swiss National Science Foundation through Project No. 200020 175750. T.T. acknowledges support from the Swiss Federal Commission for Scholarships and the former Comisión Nacional de Ciencia y Tecnología (CONICYT) now Agencia Nacional de Investigación y Desarrollo (ANID). This research was supported by NERC Grants No. NE/E003206/1 and No. NE/K003011/1 as well as EPSRC Grants No. EP/I019189/1, No. EP/K00428X/1, and No. EP/M022447/1. J.M.N.T.G. acknowledges support as a Royal Society Wolfson Research Merit Award holder (Grant No. WM150058) and as an EPSRC Established Career Fellow (Grant No. EP/M022447/1). The authors would also like to acknowledge Bob de Graffenried for his technical support and advice. 


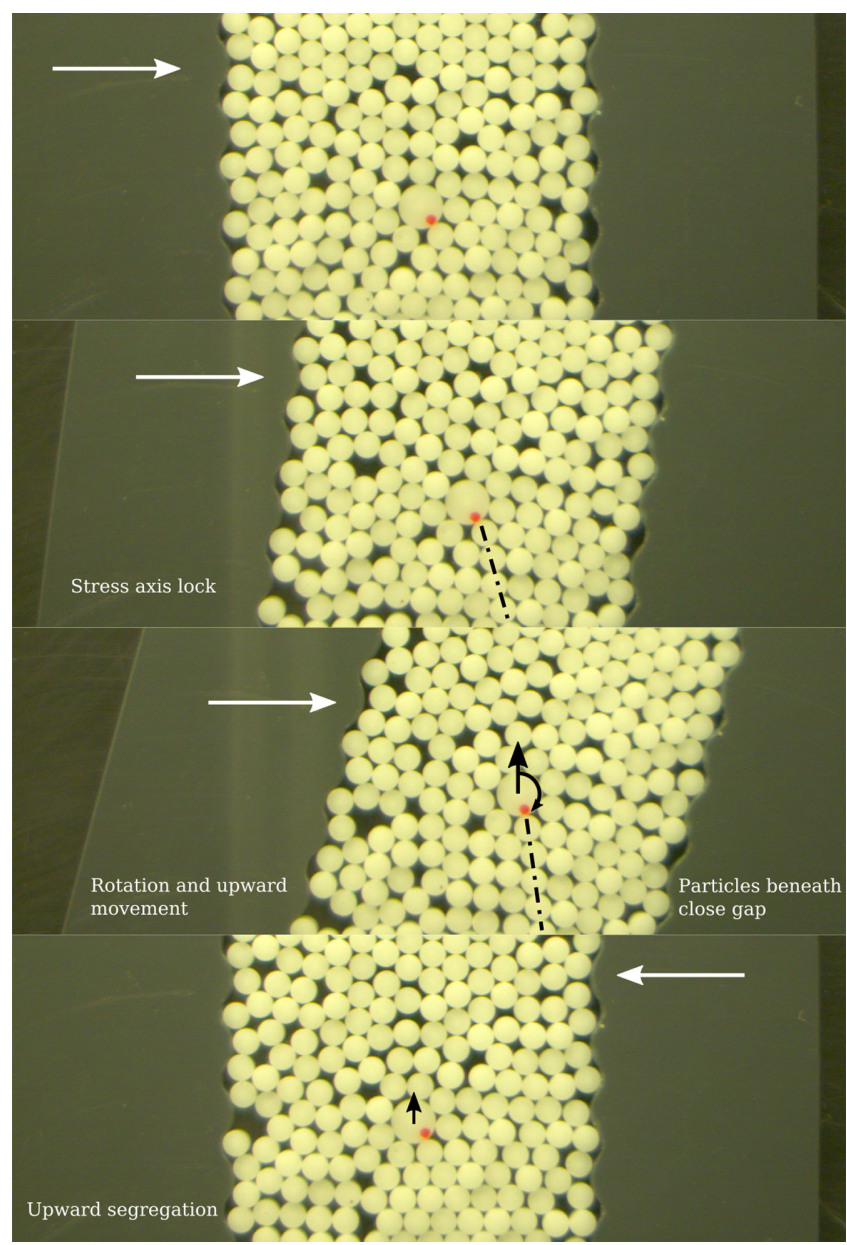

FIG. 9. Image sequence of the rotation-dominant segregation mechanism. A 10-mm intruder segregates upwards due to the interlocking of surrounding 6-mm particles that create a stress axis that locks and rotates the intruder, allowing the entrainment of the surrounding particles.

\section{APPENDIX: EXPERIMENTAL IMAGES OF THE ROTATION- AND EXPANSION-BASED SEGREGATION MECHANISMS}

To better illustrate the segregation mechanisms for large particle segregation, two image sequences from experiments are shown in this section (see Figs. 8 and 9). These images are part of the videos presented as Supplemental Material for this article [47].

[1] J. R. Johanson, Particle segregation ... and what to do about it, Chem. Eng. 85, 183 (1978).

[2] T. Shinbrot, The brazil nut effect - in reverse, Nature (London) 429, 352 (2004).

[3] E. Virčíková and L. Molnar, Recovery of copper from dump slag by a segregation process, Resour. Conserv. Recycl. 6, 133 (1992).

[4] H. A. Makse, S. Havlin, P. R. King, and H. E. Stanley, Spontaneous stratification in granular mixtures, Nature (London) 386, 379 (1997). 
[5] J. M. N. T. Gray and K. Hutter, Pattern formation in granular avalanches, Continuum Mech. Thermodyn. 9, 341 (1997).

[6] O. Pouliquen, J. Delour, and S. B. Savage, Fingering in granular flows, Nature (London) 386, 816 (1997).

[7] M. Woodhouse, A. Thornton, C. Johnson, B. Kokelaar, and J. M. N. T. Gray, Segregation-induced fingering instabilities in granular free-surface flows, J. Fluid Mech. 709, 543 (2012).

[8] J. Baker, C. Johnson, and J. M. N. T. Gray, Segregation-induced finger formation in granular free-surface flows, J. Fluid Mech. 809, 168 (2016).

[9] R. M. Iverson, The physics of debris flows, Rev. Geophys. 35, 245 (1997).

[10] G. Félix and N. Thomas, Relation between dry granular flow regimes and morphology of deposits: Formation of levées in pyroclastic deposits, Earth Planet. Sci. Lett. 221, 197 (2004).

[11] F. M. Rocha, C. G. Johnson, and J. M. N. T. Gray, Self-channelisation and levee formation in monodisperse granular flows, J. Fluid Mech. 876, 591 (2019).

[12] T. Takahashi, Debris flow, Annu. Rev. Fluid Mech. 13, 57 (1981).

[13] I. F. C. Denissen, T. Weinhart, A. Te Voortwis, S. Luding, J. M. N. T. Gray, and A. R. Thornton, Bulbous head formation in bidisperse shallow granular flow over an inclined plane, J. Fluid Mech. 866, 263 (2019).

[14] A. Mangeney, F. Bouchut, N. Thomas, J.-P. Vilotte, and M. Bristeau, Numerical modeling of selfchanneling granular flows and of their levee-channel deposits, J. Geophys. Res.: Earth Surf. 112, F02017 (2007).

[15] B. Kokelaar, R. Graham, J. M. N. T. Gray, and J. Vallance, Fine-grained linings of leveed channels facilitate runout of granular flows, Earth Planet. Sci. Lett. 385, 172 (2014).

[16] R. M. Iverson and J. W. Vallance, New views of granular mass flows, Geology 29, 115 (2001).

[17] R. Delannay, A. Valance, A. Mangeney, O. Roche, and P. Richard, Granular and particle-laden flows: From laboratory experiments to field observations, J. Phys. D 50, 053001 (2017).

[18] J. M. N. T. Gray, Particle segregation in dense granular flows, Annu. Rev. Fluid Mech. 50, 407 (2018).

[19] J. Bridgwater, Fundamental powder mixing mechanisms, Powder Technol. 15, 215 (1976).

[20] J. C. Williams, The segregation of particulate materials: a review, Powder Technol. 15, 245 (1976).

[21] A. Rosato, K. J. Strandburg, F. Prinz, and R. H. Swendsen, Why the Brazil Nuts Are on Top: Size Segregation of Particulate Matter by Shaking, Phys. Rev. Lett. 58, 1038 (1987).

[22] G. V. Middleton, Experimental studies related to problems of flysch sedimentation, in Flysch Sedimentology in North America, edited by J. Lajoie (Business and Economics Science Ltd., Toronto, 1970), pp. 253-272.

[23] S. B. Savage and C. Lun, Particle size segregation in inclined chute flow of dry cohesionless granular solids, J. Fluid Mech. 189, 311 (1988).

[24] K. M. Hill and Y. Fan, Granular temperature and segregation in dense sheared particulate mixtures, KONA Powder Part. J. 33, 150 (2016).

[25] R. P. Jones, A. B. Isner, H. Xiao, J. M. Ottino, P. B. Umbanhowar, and R. M. Lueptow, Asymmetric concentration dependence of segregation fluxes in granular flows, Phys. Rev. Fluids 3, 094304 (2018).

[26] L. A. Golick and K. E. Daniels, Mixing and segregation rates in sheared granular materials, Phys. Rev. E 80, 042301 (2009).

[27] K. van der Vaart, P. Gajjar, G. Epely-Chauvin, N. Andreini, J. M. N. T. Gray, and C. Ancey, Underlying Asymmetry within Particle Size Segregation, Phys. Rev. Lett. 114, 238001 (2015).

[28] F. Guillard, Y. Forterre, and O. Pouliquen, Scaling laws for segregation forces in dense sheared granular flows, J. Fluid Mech. 807, R1 (2016).

[29] K. van der Vaart, M. van Schrojenstein Lantman, T. Weinhart, S. Luding, C. Ancey, and A. Thornton, Segregation of large particles in dense granular flows suggests a granular Saffman effect, Phys. Rev. Fluids 3, 074303 (2018).

[30] L. Staron, Rising dynamics and lift effect in dense segregating granular flows, Phys. Fluids 30, 123303 (2018).

[31] G. Hill, S. Yeung, and S. A. Koehler, Scaling vertical drag forces in granular media, Europhys. Lett. 72, 137 (2005).

[32] L. Jing, C. Y. Kwok, and Y. F. Leung, Micromechanical Origin of Particle Size Segregation, Phys. Rev. Lett. 118, 118001 (2017). 
[33] Y. Ding, N. Gravish, and D. I. Goldman, Drag Induced Lift in Granular Media, Phys. Rev. Lett. 106, 028001 (2011).

[34] F. Guillard, Y. Forterre, and O. Pouliquen, Lift forces in granular media, Phys. Fluids 26, 043301 (2014).

[35] A. Seguin, C. Coulais, F. Martinez, Y. Bertho, and P. Gondret, Local rheological measurements in the granular flow around an intruder, Phys. Rev. E 93, 012904 (2016).

[36] T. Trewhela, C. Ancey, and J. M. N. T. Gray, An experimental scaling law for particle-size segregation in dense granular flows, J. Fluid Mech. 916, A55 (2021).

[37] A. Scott and J. Bridgwater, Self-diffusion of spherical particles in a simple shear apparatus, Powder Technol. 14, 177 (1976).

[38] D. Stephens and J. Bridgwater, The mixing and segregation of cohesionless particulate materials part II. microscopic mechanisms for particles differing in size, Powder Technol. 21, 29 (1978).

[39] A. Drescher and G. D. J. De Jong, Photoelastic verification of a mechanical model for the flow of a granular material, J. Mech. Phys. Solids 20, 337 (1972).

[40] M. Vaziri, F. Stott, and R. Spurr, Studies of the friction of polymeric materials, Wear 122, 313 (1988).

[41] H. Janssen, Versuche uber getreidedruck in silozellen, Z. Ver. Dtsch. Ing. 39, 1045 (1895).

[42] J. C. Crocker and D. G. Grier, Methods of digital video microscopy for colloidal studies, J. Colloid Interface Sci. 179, 298 (1996).

[43] P. Jop, Y. Forterre, and O. Pouliquen, A constitutive law for dense granular flows, Nature (London) 441, 727 (2006).

[44] B. Utter and R. P. Behringer, Self-diffusion in dense granular shear flows, Phys. Rev. E 69, 031308 (2004).

[45] A. Thornton, T. Weinhart, S. Luding, and O. Bokhove, Modeling of particle size segregation: Calibration using the discrete particle method, Int. J. Mod. Phys. C 23, 1240014 (2012).

[46] N. Thomas and U. D'Ortona, Evidence of reverse and intermediate size segregation in dry granular flows down a rough incline, Phys. Rev. E 97, 022903 (2018).

[47] See Supplemental Material at http://link.aps.org/supplemental/10.1103/PhysRevFluids.6.054302 for videos of the segregation mechanisms.

[48] S. Wiederseiner, N. Andreini, G. Épely-Chauvin, G. Moser, M. Monnereau, J. M. N. T. Gray, and C. Ancey, Experimental investigation into segregating granular flows down chutes, Phys. Fluids 23, 013301 (2011). 